\title{
Smart Agriculture Using Internet of Things: A Survey
}

\author{
Amera Istiqlal Badran and Manar Younis Kashmoola \\ \{amera_istiqlal@yahoo.com, manar.kashmola@uomosul.edu.iq\} \\ Computer Science Dept., College of Computer Sciences and Mathematics, University of Mosul.
}

\begin{abstract}
Internet of Things (IoT) is a new fast communication technology designed to continuously make communication among different types of devices. The researchers have exerted huge efforts to employ IoT to facilitate daily life. These include IoT big issues like securing information exchange, smart agriculture systems, and general-purpose internet access. This paper shows research efforts to enable IoT-based smart agriculture. It starts with the underlying architecture, then discusses some of the recent IoT trials. It classifies the literature by deriving a taxonomy based on technologies, communication cofactors, network types, local area wireless criteria, targets, and characteristics. Moreover, it highlights the unprecedented chances brought about by IoT-based smart architectures and their impact on human life. So IoT will solve these problems by connecting soil moisture sensors, giving us a quick response to avoid crop losses through careful monitoring and remote control of the fields. Moreover, IoT facilitates and solves most agriculture-related problems, through the use of modern technologies in monitoring plant biomarkers and controlling watering processes to increase production and efficiency. This gives us a visualization of the state of the field and enables us to take the necessary measures before the problem occurs as a way of problem anticipation.
\end{abstract}

Keywords: Internet of Things, IoT Protocols, IoT Application, Agriculture, Agriculture IoT.

\section{Introduction}

IoT allows the possibility of connecting various things through the internet and enables people to communicate and interact with the things they use and deal with in their daily lives the way they like. It provides service without the need to be in a specific place. IoT aims to create a better life for human beings by providing new humanitarian services that could not have been implemented previously [1].

As with the internet, the IoT network does not rely on fixed architecture, but modern concepts, technologies and applications which all push the IoT network into a dynamic and constantly changing format and architecture [1],[2].

However, the IoT network can be viewed through the type of many things. Things can interact with each other on the web without human intervention or human interaction. The IoT network can also be viewed through its sensors, microchips, operating systems, wired and wireless networks, in addition to the massive data storage in cloud-based data stores and applications. The evolution of internet protocols indicates the emergence of a new generation of protocols that contribute to the development of IoT [1]. 
The IoT works by connecting sensors through microchips, microcontrollers, wired and wireless networking, and access to a large data storage, allowing something to interact with something else or human interacted with something [3],[4]. The IoT nodes that are configured to connect objects can provide data for access to and authorization of shared cloud resources to collect and extract data and make choices by investigating the data collected. The emergence of the IoT has led to broad communication among people, devices and things. Many different IoT devices are connected in many applications from smart grids to smart agriculture [5],[6]. Recently, investments have increased globally in the field of cloud computing and cloud storage, which in turn greatly supports the idea of IoT and connect all the devices of the world to the internet and access their data cloud. To create smart agriculture, for example, we will actually need to store data in the cloud so that things and users can also access and manipulate data anytime, anywhere, without having to know how to implement services [5],[7].

Reports illustrate the predictability of increasing the number of internet-connected devices twofold from 22.9 billion in 2016 to more than 50 billion by 2020 as shown in Figure 1 [8].

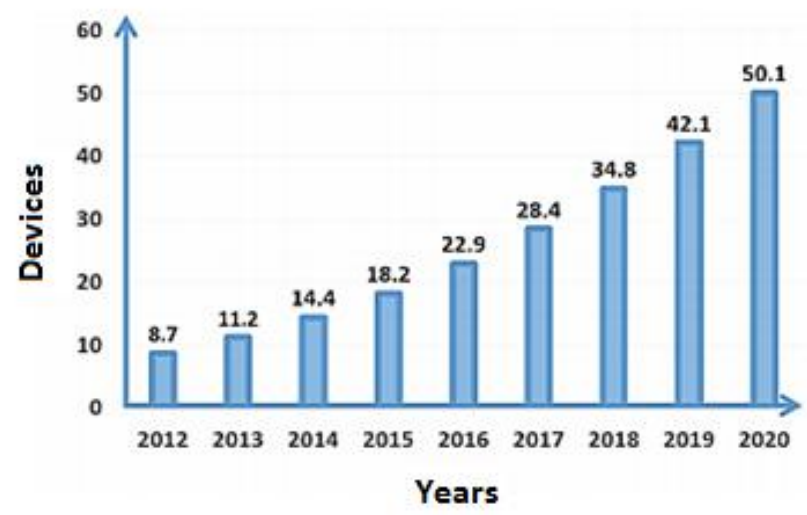

Fig. 1. The Sum of Internet-Connected Devices [9].

To obtain soil moisture in the near-surface layer, a moisture sensor is needed. The soil moisture obtained by the nodes is then classified, and the primary moisture content is obtained. Zigbee WSN technology is used, as well as low cost and low power consumption. Artificial intelligence (AI) is integrated with the development of agriculture and thus obtaining smart agriculture. [6].

Security problem has become one of the most important challenges in the world of things such as access control, secure storage of data and secure communications. This reflects important challenges in the development of the IoT [10]. Therefore, each new sensor or another device that will be used in the IoT application should be tested and secured.

This paper is prepared as follows: Section 2: Sensors used for detection; Section 3: Data analysis in IoT application; Section 4: Agricultural problems; Section 5: IoT based monitoring system in Smart Agriculture; Section 6: Plant factory based on IoT; Section 7: Applications of agricultural 
information; Section 8: closes the paper with some concluding remarks.

\section{Sensors Used for Detection}

The sensors used in Figure 2. are intended to monitor the field of the environment such as agriculture, for example, sensors such as MQ4 and MQ7 are used for natural gas and carbon monoxide sensing respectively [11]. DHT11 is used to monitor the temperature and humidity of the environment. The soil moisture sensors are used to measure the moisture of the soil and continuously monitor it to follow the watering of the fields. While Esp8266 is a Wi-Fi module that helps communicate between the previously mentioned sensors and the users' computers [5],[12].

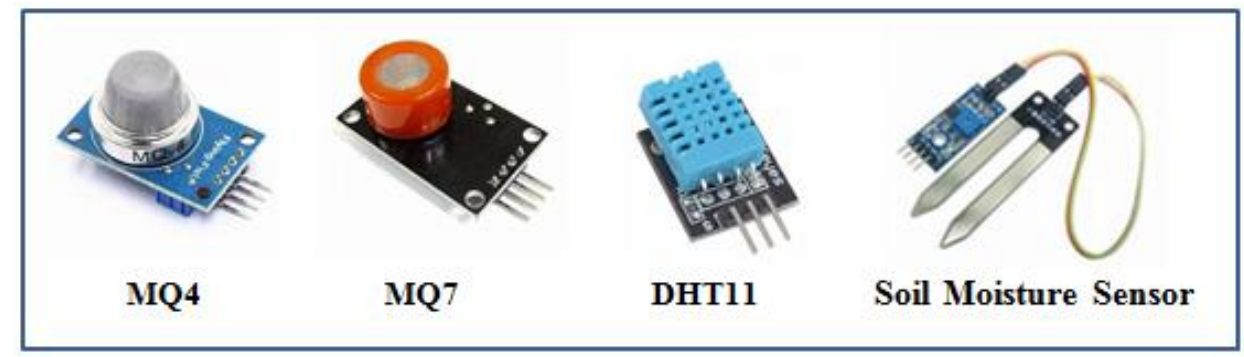

Fig. 2. Different Types of Sensors [13].

Protocols are important elements of the IoT that support and enable things to work. Since the protocol is the language of understanding among computers, it includes a set of rules and laws governing a particular process in the field of IoT. If there is any difference in the types of protocols among computers, data will not be transmitted properly among them because of the lack of recognition of orders by the parties concerned. Several major IoT protocols are often used in the process of communication among smart internet devices. These protocols have different structures according to their needs and functionality. Selecting the appropriate protocol for implementation will lead to having improvements in the areas of internet applications such as smart agriculture. Here are some IoT protocols [14],[15],[16]:

- The Data Distribution Service (DDS).

- The Advanced Message Queuing Protocol (AMQP).

- The Message Queue Telemetry Transport (MQTT).

- The Secure MQTT (SMQTT).

- The MQTT- Sensor Networks (MQTT-SN).

- The Constrained Application Protocol (CoAP).

- The Extensible Messaging and Presence Protocol (XMPP). 


\section{Data Analysis in IoT Applications}

We should know that the huge increase in the number of devices connected to the IoT leads to a doubling of data growth completely with the growth of the IoT. The big data sources result from the application of IoT. In smart agriculture, for example, collecting and analyzing data from devices, sensing and understanding of data on the IoT were used in order to avoid equipment breakdowns in advance, improve plant crops and do marketing. Researchers have examined the challenges connected with the successful spread of IoT and big data. So, the convergence of these studies creates many chances for data prosperity and analysis in the field of IoT. These developments and the increase in data need major requirements to manage big data and to enable analysis in the IoT environment [17],[18]. Table 1. reviews the advantages of big data and analytics in IoT applications.

Table 1. Advantages of Data Analytics for IoT Applications[17].

\begin{tabular}{|l|l|}
\hline \multicolumn{1}{|c|}{ IoT Applications } & \multicolumn{1}{|c|}{ Data Analysis } \\
\hline Smart Transportation & $\begin{array}{l}\text { (a) Decreases the number of accidents by looking into their history. } \\
\text { (b) Minimizes traffic bottleneck. } \\
\text { (c) Optimizes delivery movements. } \\
\text { (d) Ensures road safety. }\end{array}$ \\
\hline Smart Healthcare & $\begin{array}{l}\text { (a) Expects epidemics, cures, and illness. } \\
\text { (b) Helps insurance companies make better policies. } \\
\text { (c) Picks up the warning signs of any severe illnesses during their early stages. }\end{array}$ \\
\hline Smart Grid & $\begin{array}{l}\text { (a) Helps designing an optimal pricing plan according to the current power } \\
\text { (b) Expects future supply needs. } \\
\text { (c) Ensures a suitable level of electricity supply. }\end{array}$ \\
\hline Smart Inventory & $\begin{array}{l}\text { (a) Detects deceitful cases. } \\
\text { (b) Deliberately places an advertisement. } \\
\text { (c) Understands customer needs. } \\
\text { (d) Identifies possible risks. }\end{array}$ \\
\hline $\begin{array}{l}\text { Smart Agriculture } \\
\text { [19],[20],[21] }\end{array}$ & $\begin{array}{l}\text { (a) Provides nutrients to the soil if they are depleted. } \\
\text { (b) Implements improved inheritance. } \\
\text { (c) Increases economic development, protects the environment and deals with } \\
\text { (d) Stimalates plant growth. } \\
\text { (e) Increases security and reduces cost. }\end{array}$ \\
\hline
\end{tabular}

\section{Agricultural Problems}

In the field of agriculture, there are many problems and challenges. Such of these are: the spread of diseases and agricultural pests, controlling difficulty, improperly treated soil, inadequate drainage and irrigation, the inability to manage weeds, the lack of food for the soil if depleted and many other problems. These problems lead to the loss of a large number of crops as well as environmental risks 
due to excessive use of chemicals. Numerous studies have been conducted to address and eliminate these problems. One of the most important solutions is to use the field of AI to solve the problems of agriculture. Systems are being developed to help agricultural experts find solutions [13],[19]. As studies continued, these problems were solved using the IoT, where smart agriculture played a major role in solving them [22].

The continuous increase in global food demand enables the Internet to open new horizons in this field. Smart irrigation systems are used to increase production and combat agricultural pests. Intelligent irrigation systems reduce the amount of water spent in the irrigation process, which reduces soil erosion for the agricultural field. Consequently, the field can be monitored to measure the vital indicators of plants such as temperature, soil moisture, and PH for water and soil. IoT can enable monitoring the field and controlling agricultural pests. This is carried out through knowing the cause by measuring plant vital signs, which are taken by sensors. Smart irrigation systems are built using electronic chips such as ESP8266 and other sensors such as soil moisture sensors to measure the quality of watering and control this process [22],[23].

\section{Smart Agriculture IoT-Based Monitoring System}

Recent technological advances in the world have led to increased production, as well as significantly improved production efficiency. Through the use and development of agricultural machinery, that can be operated both automatically and manually, it can be used for different agricultural activities such as: spraying, cutting, weeding, plowing and other works. Moreover, using the controller to monitor air temperature and humidity; soil moisture, besides field water management. This smart technology has had a significant impact on increasing and improving production [23].

\section{Plant Factory Based on IoT}

The concept of IoT is related to the modern agricultural field, which consists of a soil-free environment, such as soil solution control technology. The IoT technology affects both soil solution, photosynthesis, carbon dioxide, atmospheric humidity and wind pressure by affecting soil moisture. All of the above mentioned comes under the name of intelligent irrigation technology. Internet technology affects agricultural production by affecting plant cell manufacturing. The production of the plant cell is highly efficient in the agricultural system through the control of the IoT on temperature, humidity and carbon dioxide concentration. Also, the IoT reduces the cost of production by reducing labor, especially for crops that need special conditions. The IoT serves the plant cell production through sensors, reliable delivery and intelligent handling, as well as intelligent farming control architecture based on cloud computing with IoT [22],[24],[25].

\section{IoT Applications in Agriculture}

The world population is increasing and may reach 9.6 billion by 2050 . With this increase in population, the demand for agriculture will increase to increase food production. The IoT is capable of transforming the field of agriculture into intelligent agriculture. This is done by addressing the challenges facing the agricultural environment in order to increase the production of agriculture to meet the needs of the population. This is achieved through the reduction of manpower and the use 
of fertilizers to increase and improve production[26].

Keshtgari, M. \& Deljoo, A..(2011)[6] proposed the uses of the Zigbee technology in the field of agriculture and the acquisition of agricultural data after using climate sensors. The main station communicates with other stations and collects local information, then data processing takes place to make decisions and this process is done on large areas of agriculture in order to improve the quality of agricultural production with precise irrigation. Cost reduction and the use of Zigbee technology enable real-time field monitoring, as well as automatic irrigation control and remote operation of machines in the field. Zigbee technology has been used because it is a short-range, low-energy wireless network suitable for use in remote and isolated areas such as agricultural fields.

One of the adaptive techniques used to solve farming problems is the artificial neural network (ANN), and this technique finds many ways to analyze complex data [27]. Hennell, eh.C. \& others (2010)[20] proposed neural drip irrigation systems where ANNs were developed for the system to function properly. They predicted water distribution in the field.

Prakash, C., Rathor, A.S. \& Thakur, G.S.M., (2013)[28] proposed designing a system for the soybean crop. They introduced a typical expert system of soybeans called Prithvi. Fuzzy logic was used in this system so that the expert is the farmer who will be the decision-maker. The main objective of this expert system is to develop the expert farmers and help them to improve and increase the production of the soybean crop, by proposing the period and method of sowing and the choice of fertilizers.

Zografos, A. (2014) [21] introduced a small farming system, designed, built and evaluated to provide farmers with useful data on soil, water supply and general conditions of their fields in an easy-to-use manner. This system aims to make agriculture and irrigation more efficient to make the farmer able to make the right and appropriate decisions, and thus save time and effort and reduce costs. Location diversity and climate have an impact on agriculture. Other environmental standards over time, make farmers' decision-making more complex and require additional empirical knowledge. The application of wireless sensor networks to monitor environmental factors and the combination of this information with a user-specific web service enables farmers to effectively exploit their knowledge to extract the best results from their agriculture.

Madushanki, A. R., \& others (2019)[29] used sophisticated internet-related agricultural applications, taking sensor data from 60 scientific papers published between 2016 and 2018 to make appropriate measurements. The results from previous studies have shown that smart agriculture is of great importance in water, crop, livestock, and irrigation management. Concerning the collection of sensor data, the highest results were recorded when measuring temperature and humidity. There are also sensors for soil moisture and the degree of soil reaction.

\section{Conclusion}

Through this paper, a comprehensive survey of IoT applications has been conducted in all aspects of our agricultural life. Smart agriculture is created using agricultural information based on IoT and cloud computing. It is found that the application of IoT in agriculture has many advantages, such as: maintaining and improving agricultural local production, significantly improving resource 
efficiency by dealing with a large amount of data obtained, having full control at the lowest costs and losses in agricultural reality, controlling watering processes automatically, determining the quantities of watering and controlling soil erosion through decreasing water wasting, preventing the emergence of agricultural pests, and overcoming previous traditional reliance on farmers, which makes this process tedious, tiring, and useless. As for the disadvantages, relying on IoT in agriculture leads to higher percentages of unemployment, in addition to some negative psychological effects on the continuous use of smart technology instead of human self-abilities.

\section{References}

[1] Sethi, P., \& Sarangi, S. R. (2017). "Internet of things: architectures, protocols, and applications". Journal of Electrical and Computer Engineering.

[2] Khan, R., Khan, S. U., Zaheer, R., \& Khan, S. (2012). "Future internet: the internet of things architecture, possible applications and key challenges". In 2012 10th international conference on frontiers of information technology (pp. 257-260). IEEE.

[3] Chifor, B. C., Bica, I., Patriciu, V. V., \& Pop, F. (2018). "A security authorization scheme for smart home Internet of Things devices". Future Generation Computer Systems, 86,p 740-749.

[4] Conti, M., Dehghantanha, A., Franke, K., \& Watson, S. (2018). "Internet of Things security and forensics: Challenges and opportunities".

[5] Jyostsna Vanaja, K., Aala Suresh, S., Srilatha, K., Vijay Kumar, M. and Bharath, M..(2018). "IoT based Agriculture System Using NodeMCU". International Research Journal of Engineering and Technology (IRJET). Volume: 05 Issue: 03.

[6] Keshtgari, M. \& Deljoo, A. (2011). "A Wireless Sensor Network Solution for Precision Agriculture Based on ZigBee Technology". doi:10.4236/wsn. 41004 Published Online (http://www.SciRP.org/journal/wsn).

[7] Mai, V., \& Khalil, I. (2017). "Design and implementation of a secure cloud-based billing model for smart meters as an Internet of things using homomorphic cryptography". Future Generation Computer Systems, 72, 327-338.

[8] Ahmed, E., Yaqoob, I., Hashem, I. A. T., Khan, I., Ahmed, A. I. A., Imran, M., \& Vasilakos, A. V. (2017). "The role of big data analytics in Internet of Things". Computer Networks, 129, 459-471.

[9] Burhan, M., Rehman, R., Khan, B., \& Kim, B. S. (2018). "IoT elements, layered architectures and security issues: A comprehensive survey". Sensors, 18(9), 2796.

[10] Zarpelao, B. B., Miani, R. S., Kawakani, C. T., \& de Alvarenga, S. C. (2017). "A survey of intrusion detection in Internet of Things". Journal of Network and Computer Applications, 84, 25-37.

[11] Babu, K. S., \& Nagaraja, C. (2018). "Calibration of MQ-7 and Detection of Hazardous Carbon Monooxide Concentration in Test Canister". international journal of advanced research ISSN: 2454-132X Impact factor: 4.295 (Volume 4, Issue 1).

[12] Lopez, J., Rios, R., Bao, F., \& Wang, G. (2017). "Evolving privacy: From sensors to the Internet of Things". Future Generation Computer Systems, 75, 46-57.

[13] Jha, K., Doshi, A., Patel, P., \& Shah, M. (2019). "A comprehensive review on automation in agriculture using artificial intelligence". Artificial Intelligence in Agriculture. 
[14] Fysarakis, K., Askoxylakis, I., Soultatos, O., Papaefstathiou, I., Manifavas, C., \& Katos, V. (2016). "Which IoT protocol? comparing standardized approaches over a common m2m application". In 2016 IEEE Global Communications Conference (GLOBECOM) (pp. 1-7). IEEE.

[15] Ponnusamy, K., \& Rajagopalan, N. (2018). "Internet of Things: A Survey on IoT Protocol Standards". In Progress in Advanced Computing and Intelligent Engineering (pp. 651-663). Springer, Singapore.

[16] Salman, T., \& Jain, R. (2015). "Networking protocols and standards for internet of things". Internet of Things and Data Analytics Handbook , 7. P.14-18.

[17] Bessis, N., \& Dobre, C. (Eds.). (2014). "Big data and internet of things: a roadmap for smart environments". (Vol. 546). Basel, Switzerland: Springer International Publishing.

[18] Hashem, I. A. T., Chang, V., Anuar, N. B., Adewole, K., Yaqoob, I., Gani, A., ... \& Chiroma, H. (2016). "The role of big data in smart city". International Journal of Information Management, 36(5), 748-758.

[19] Bannerjee, G., Sarkar, U., Das, S., \& Ghosh, I. (2018). "Artificial Intelligence in Agriculture: A Literature Survey". International Journal of Scientific Research in Computer Science Applications and Management Studies., 7(3), p 1-6.

[20] Hinnell, A. C., Lazarovitch, N., Furman, A., Poulton, M., \& Warrick, A. W. (2010). "Neuro-Drip: estimation of subsurface wetting patterns for drip irrigation using neural networks". Irrigation science, 28(6), 535-544.

[21] Zografos, A. (2014). "Wireless Sensor-based Agricultural Monitoring System". School of Information and Communication Technology (ICT) KTH Royal Institute of Technology.

[22] Nayyar, A., \& Puri, E. V. (2016). "Smart Farming: IoT Based Smart Sensors Agriculture Stick for Live Temprature and Moisture Monitoring using Arduino Cloud Computing \& Solar Technology". In Conference: The International Conference on Communication and Computing Systems (ICCCS).

[23] Veena, S., Mahesh, K., Rajesh, M., \& Salmon, S. (2018). "The Survey on Smart Agriculture Using IoT". International Journal of Innovative Research in Engineering \& Management (IJIREM) ISSN: 2350-0557, Volume-5, Issue-2.

[24] Hong, L. (2011). "IoT and cloud computing: Advance Strategic New Industry"[M]. Beijing, Posts \& Telecom Press, China.

[25] Xing, Y., \& Zhan, Y. (2012). "Virtualization and cloud computing". In Future Wireless Networks and Information Systems (pp. 305-312). Springer, Berlin, Heidelberg.

[26] Mohammed Khaleel uddin. N, Pratap. K, Vineeth. K \& Mrs. Maheswari. M. (2018). "Warehouse Management using IoT". International Research Journal of Engineering and Technology (IRJET) Volume: 05 Issue: 04.

[27] Arif, C., Mizoguchi, M., \& Setiawan, B. I. (2013). "Estimation of soil moisture in paddy field using artificial neural networks". International Journal of Advanced Research in Artificial Intelligence arXiv preprint arXiv:1303.1868.

[28] Prakash, C., Rathor, A. S., \& Thakur, G. S. M. (2013). "Fuzzy based Agriculture expert system for Soyabean". In International Conference on Computing Sciences WILKES100-ICCS2013, Jalandhar, Punjab, India.

[29] Madushanki, A. R., Halgamuge, M. N., Wirasagoda, W. S., \& Syed, A. (2019). "Adoption of the Internet of Things (IoT) in Agriculture and Smart Farming towards Urban Greening: A Review". International Journal of Advanced Computer Science and Applications, 10(4), 11-28. Vol. 10. 